PHYSICAL REVIEW D 95, 069901(E) (2017)

\title{
Publisher's Note: Results from the first use of low radioactivity argon in a dark matter search [Phys. Rev. D 93, 081101(R) (2016)]
}

\author{
P. Agnes et al. \\ (DarkSide Collaboration)
}

(Received 6 February 2017; published 8 March 2017)

DOI: 10.1103/PhysRevD.95.069901

This paper was published online on 8 April 2016 with an omission of two authors in the author list and an incomplete Acknowledgment section. J. Hall and P. H. Humble and their affiliation "Pacific Northwest National Laboratory, Richland, Washington 99352, USA" have been added to the author list. The Acknowledgments on page 5 should read as "The DarkSide-50 Collaboration would like to thank LNGS and its staff for invaluable technical and logistical support. This report is based upon work supported by the U.S. National Science Foundation (NSF) (Grants No. PHY-0919363, No. PHY1004072, No. PHY-1004054, No. PHY-1242585, No. PHY-1314483, No. PHY-1314507 and associated collaborative grants, No. PHY-1211308, and No. PHY-1455351), the Italian Istituto Nazionale di Fisica Nucleare, the U.S. Department of Energy (Contracts No. DE-FG02-91ER40671, No. DE-AC02-07CH11359, and No. DE-AC05-76RL01830), and the Polish NCN (Grant No. UMO-2012/05/E/ST2/02333). We thank staff at the Pacific Northwest National Laboratory, particularly Dr. C.E. Aalseth, Mr. M.E. Panisko, and Dr. R. M. Williams, for their help in the processing of the underground argon. We thank the staff of the Fermilab Particle Physics, Scientific and Core Computing Divisions for their support. We acknowledge the financial support from the UnivEarthS Labex program of Sorbonne Paris Cité (ANR-10LABX-0023 and ANR-11-IDEX-0005-02) and from the São Paulo Research Foundation (FAPESP)." The paper has been corrected as of 16 February 2017. The author list and Acknowledgments are incorrect in the printed version of the journal. 